\title{
UTILIDAD Y NECESIDAD DEL ANDRÓLOGO EN LAS UNIDADES DE REPRODUCCIÓN ASISTIDA
}

\author{
I. GALMÉS BELMONTE \\ Unidad de Andrología. GINEFIV. Madrid. \\ Actas Urol Esp. 28 (5): 364-376, 2004
}

\section{RESUMEN}

UTILIDAD Y NECESIDAD DEL ANDRÓLOGO EN LAS UNIDADES DE REPRODUCCIÓN ASISTIDA

En el presente trabajo se recoge la experiencia del autor relacionada con el estudio y tratamiento de la patología del varón en el seno de una unidad de reproducción asistida. Se hace una revisión de los protocolos de estudio, las causas de la esterilidad masculina, los posibles tratamientos etiológicos, la participación del andrólogo en la recuperación quirúrgica de espermatozoides y la valoración o "screening" previa a cualquier ciclo de FIV o FIV-ICSI.

Como conclusiones, podemos afirmar que aplicando un adecuado protocolo de estudio es posible llegar al diagnóstico etiológico de la esterilidad masculina en un 78,8\% de los casos, e instaurar un tratamiento específico en casi la mitad de los varones $(47,8 \%)$. Los resultados conseguidos con dichos tratamientos, haciendo una selección previa de los casos a tratar, pueden igualar e incluso superar a los ofrecidos por las técnicas de reproducción asistida. Los mejores resultados, en porcentaje de embarazos, se han conseguido con las vaso-vasostomías y con la cirugía desobstructiva de los conductos eyaculadores. Por otra parte, también resulta aconsejable el estudio de posibles alteraciones genéticas y factores de mal pronóstico en aquellos varones cuyas parejas vayan a someterse a ciclos de FIV-ICSI.

PALABRAS CLAVE: Esterilidad masculina: etiología y tratamiento. Vaso-vasostomía. Obstrucción de conductos eyaculadores. Patología genética del varón.

\section{ABSTRACT \\ USEFULNESS AND NECESITY OF THE ANDROLOGIST IN ASSISTED REPRODUCTIONS UNITS}

In this issue we make a review of our experience in the management of the sterile men: protocols used for the study of men's sterility, causes and specific treatments, surgical recovery of spermatozoa and screening before IVF or IVF-ICSI.

We concluded that with an adequate protocol, it's possible to establish the aetiology of men's infertility in a $78.8 \%$ of the cases and it's also possible to initiate a specific treatment in almost a $47.8 \%$ of men. Results of specific treatment, with a previous selection of patients, could be better than those of assisted reproduction techniques. Best results are obtained in cases of vaso-vasostomy (vasectomy reversal) and obstruction of ejaculatory duct. It's also needed a study of genetics causes and bad prognostic circumstances in those men whose partner is going on with an IVF or IVF-ICSI cycle.

KEY WORD: Man sterility: etiology and treatment. Vaso-vasostomy. Eyaculatory duct obstruction. Genetic pathology of man. 


\section{INTRODUCCIÓN}

El factor masculino participa en la etiología de la esterilidad conyugal en un elevado porcentaje de casos. La mayor parte de los trabajos publicados reconocen que dicho porcentaje supera el $65 \%{ }^{1-3}$. De una manera aproximada podríamos afirmar que en el varón se encuentra la causa de la esterilidad de la pareja en un tercio de los casos como factor aislado y en otro tercio junto a un factor femenino también alterado. Este elevado porcentaje de casos hace cada vez más necesaria la participación del andrólogo en las Unidades de Reproducción Asistida (URA).

Desde nuestra perspectiva, su participación debería centrarse en los siguientes aspectos:

- Diagnóstico y tratamiento etiológico del factor masculino en todos aquellos casos en los que sea posible.

- Recuperación de espermatozoides en varones con azoospermia o necrozoospermia.

- Screening andrológico en parejas que se van a someter a FIV-ICSI.

Ignorar el factor masculino en sus tres aspectos anteriormente enumerados, recurriendo exclusivamente al andrólogo para recuperación de espermatozoides, creemos es absolutamente incorrecto desde varios puntos de vista:

- Ético y deontológico: no se está solucionando el problema

médico que presenta el varón, circunstancia que resulta posible en un elevado porcentaje de $\operatorname{casos}^{4-7}$.

- Legal: el prescindir del screening andrológico previo a FIV-ICSI podría hacernos incurrir en responsabilidad legal, puesto que no se estudian posibles problemas genéticos trasmisibles ni factores capaces de reducir las posibilidades de éxito de las Técnicas de Reproducción Asistida (TRA) ${ }^{8-10}$.

\section{ESTUDIO DEL FACTOR MASCULINO: DIAGNÓSTICO Y TRATAMIENTO ESPECÍFICO}

\subsection{Valoración inicial de las parejas con esterilidad}

La valoración inicial del factor masculino en casos de esterilidad conyugal resulta muy sencilla, consiste en la realización de una breve historia clínica y en un estudio del semen. Nosotros consideramos necesario analizar al menos 2 seminogramas del varón debido a la gran variabilidad individual que es posible encontrar en los resultados. En todos los casos se confirmará el cumplimiento estricto de las normas de recogida de la muestra y la ausencia de procesos que pudieran alterar los resultados del estudio.

Las indicaciones para iniciar un estudio andrológico más completo serían las siguientes:

- Alteración seminal de cualquier naturaleza.

- Abortos de repetición en la pareja (3 o más).

- Antecedentes de enfermedad genética del varón o de la familia.

- Fracaso de 3 o más ciclos previos de FIV.

- Alteración de la eyaculación o de la erección que impida el coito o la fecundación normal.

\subsection{Protocolo de estudio del varón}

En las Figuras 1, 2 y 3, y Tablas I y II se recogen los protocolos de estudio utilizados en nuestra Unidad de Reproducción para cada una de las situaciones anteriormente enumeradas. Las pruebas diagnósticas utilizadas se enumeran a continuación.

- Estudio nivel I (valoración inicial):

- Historia clínica básica: antecedentes de alteraciones de la erección o eyaculación, antecedentes de enfermedad genética o familiar, etc.

- Seminogramas (al menos 2).

- Estudio nivel II (valoración básica):

- Historia clínica y exploración física completa, especial atención a aspectos específicos andrológicos.

- Determinación de Anticuerpos Anti-espermatozoides (AAE).

- Perfil hormonal: FSH, LH y Testosterona (Prolactina si existe disminución de la líbido).

- Ecografia testicular bilateral ${ }^{11}$.

- Estudio nivel III (valoración específica, dependiendo de las alteraciones encontradas):

- Ecografia doppler testicular: en casos de varicocele clínico (descartar reflujo venoso derecho) o dudoso.

- Ecografia transrectal de próstata y vesículas seminales en casos de sospecha de obstrucción de eyaculadores o patología próstatoseminal $^{12}$. 


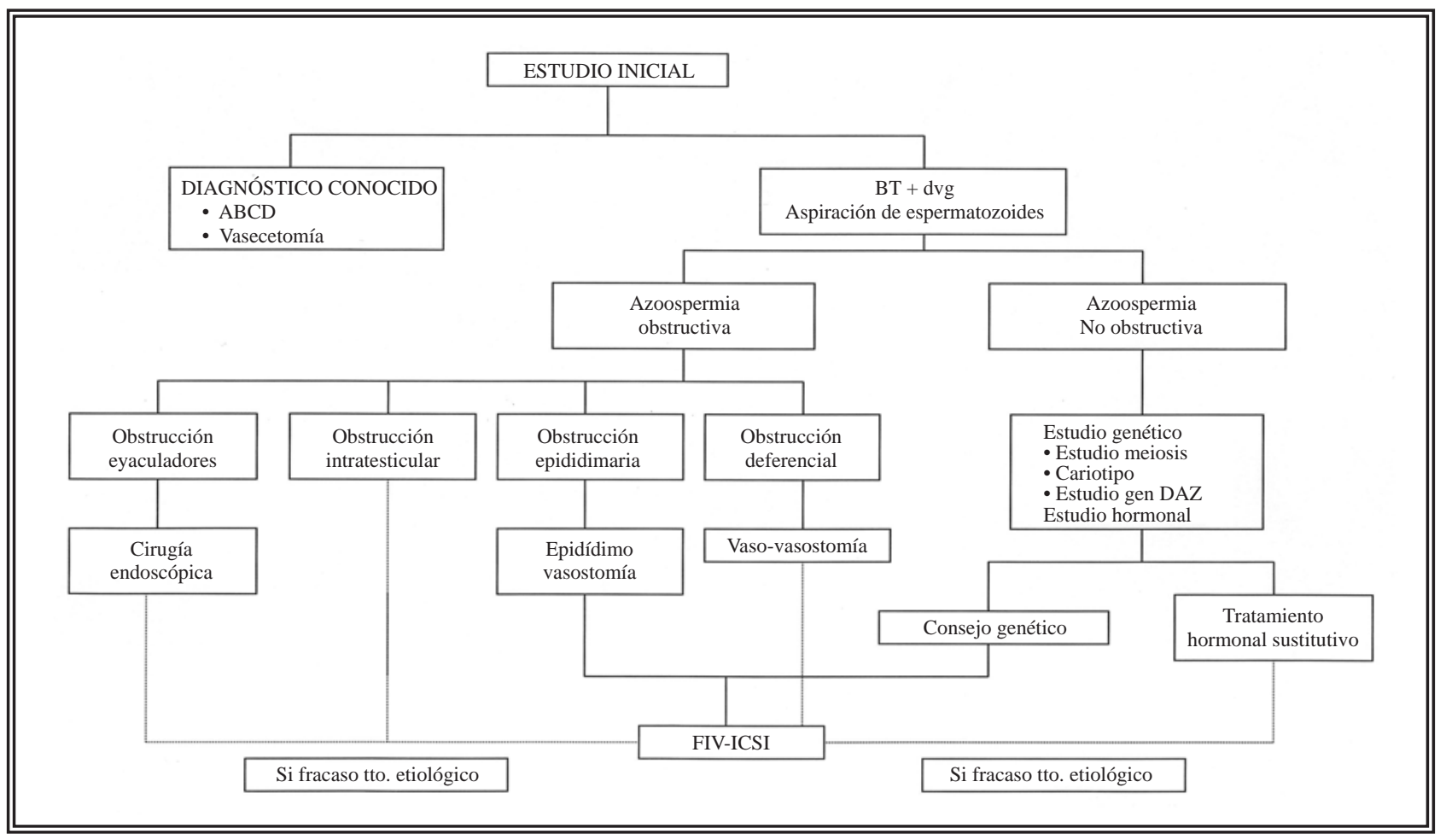

FIGURA 1. Protocolo de estudio de las azoospermias

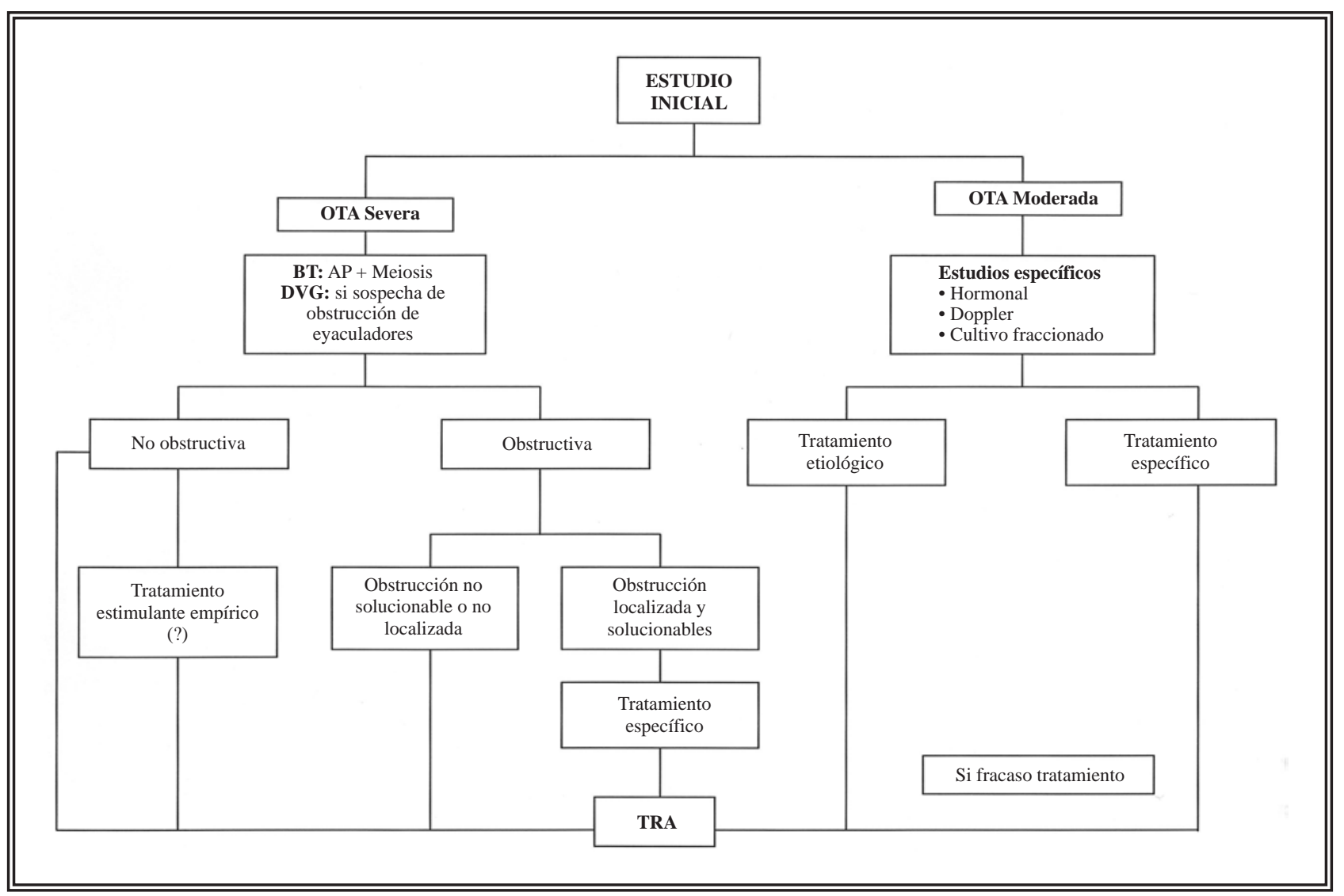

FIGURA 2. Protocolo de estudio de las oligozoospermias 


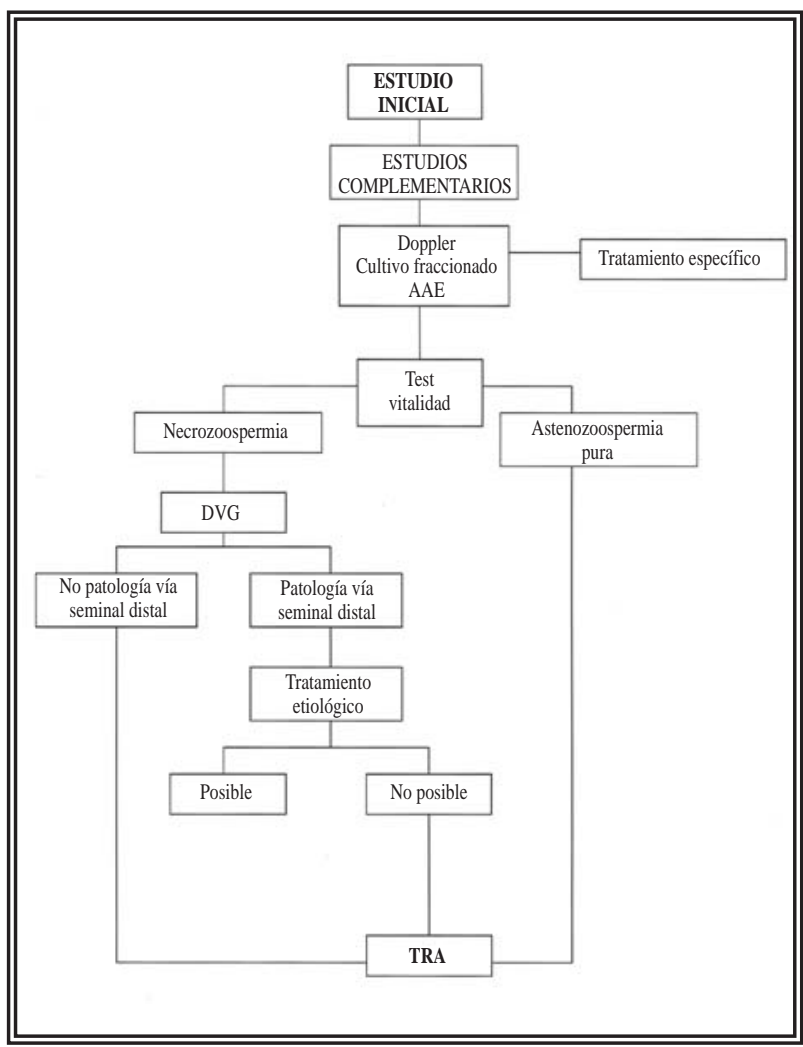

FIGURA 3. Protocolo de la astenozoospermia.

- Cultivo fraccionado de orina y semen, en casos de sospecha de infección de la vía seminal $^{13}$.

- Estudio de orina post-eyaculación en casos de sospecha de eyaculación retrógrada ${ }^{14}$.

- Cariotipo en sangre periférica en casos de azoospermia u OTA severa.

- Determinación de delecciones en el cromosoma $\mathrm{Y}$ en casos de azoospermia u OTA severa.

- Determinación de mutaciones del gen de la Fibrosis Quística en casos de agenesia uni o bilateral de conductos deferentes o antecedentes familiares de FQ.

- Deferento-vesiculografía: en caso de sospecha de patología obstructiva de la vía seminal.

- Estudio citogenético de meiosis en casos de azoospermia u OTA severa, fracaso de varios ciclos de FIV previos, abortos de repetición, etc.

- FISH de espermatozoides en casos de azoospermia u OTA severa, fracaso de varios ciclos de FIV previos, abortos de repetición, etc. ${ }^{15}$.

\section{TABLA I}

PROTOCOLO DE ESTUDIO DE LOS ABORTOS DE REPETICIÓN

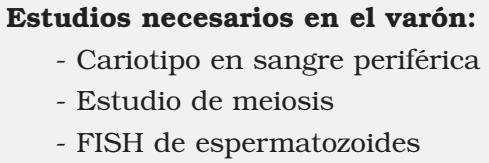

En caso de alteración se recomienda:

- Consejo genético: evaluar riesgo para la pareja

Diagnóstico preimplantacional (si preciso)

Amniocentesis

\section{TABLA II}

PROTOCOLO DE ESTUDIO EN CASO DE FRACASO DE 3 O MÁS CICLOS DE FIV

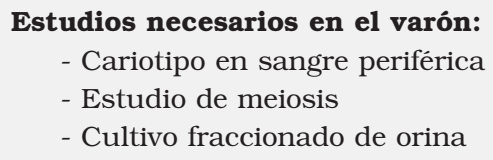

En caso de cariotipo o estudio de meiosis alterado: - Consejo genético

- Diagnóstico preimplantacional

En caso de cultivo fraccionado de orina positivo: - Tratamiento antibiótico según antibiograma

- Normas para recogida muestra de semen - Si no se consigue erradicar la infección: tratamiento profiláctio antes de recoger la muestra de semen

- Biopsia testicular, estudio histológico ${ }^{16,17}$.

- Estudio de la capacidad eréctil, estudio de las alteraciones de la eyaculación

\subsection{Clasificación etiológica}

Aplicando un adecuado protocolo de estudio, es posible llegar al diagnóstico etiológico en 4/5 partes de los varones estudiados $(78,8 \%$ en nuestra serie). Una vez establecido éste, será posible plantear un tratamiento oportuno en un gran porcentaje de casos (del 47,8\% en nuestro caso). En la Tabla III se han recogido las etiologias encontradas en los varones estudiados en nuestra Unidad de Andrología.

Tras haber establecido la causa del factor masculino, habrá que plantearse su posible solución y las probabilidades reales de mejorar la calidad seminal en cada caso. Esta posibilidad va 


\section{TABLA III}

CAUSAS DE LA ALTERACIÓN SEMINAL DEL VARÓN O MOTIVO DE ESTUDIO EN NUESTRA UNIDAD DE ANDROLOGÍA

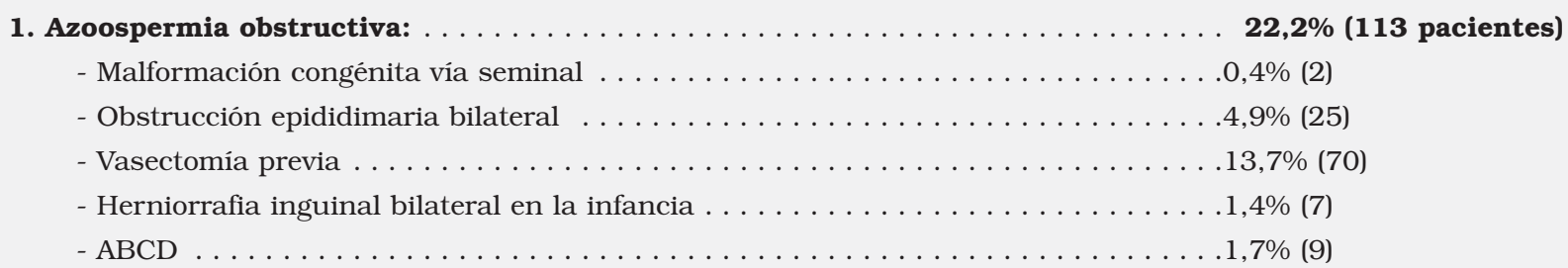

a depender no sólo de la etiología, sino también del grado de deterioro que ésta haya provocado en el epitelio germinal (severidad de la lesión y reversibilidad de la misma). Cuando un factor causal actúa durante un período prolongado de tiempo, frecuentemente llega a provocar lesiones muy severas e irreversibles sobre el epitelio germinal. En estas situaciones no se conseguirá mejorar la calidad seminal a pesar de que se elimine aquel.

En aquellas situaciones en las que se sospeche un daño testicular severo e irreversible (calidad seminal muy deteriorada, testículos atróficos en la exploración física, FSH elevada, estructura ecográfica testicular muy dishomogénea, etc.) antes de proceder a cualquier tratamiento, se debería confirmar la capacidad productiva residual del testículo, descartando los casos con deterioro severo o irreversible ${ }^{18}$. La adecuada selección de los pacientes, identificando aquellos con claras posibilidades de mejoría tras la instauración de los tratamientos específicos, resulta clave para obtener resultados satisfactorios.

Por otra parte, no se debe perder de vista que los procedimientos de fecundación "in vitro" (FIV) están consiguiendo unos porcentajes de éxito de entre 25-40\% por ciclo (dependiendo de los casos) ${ }^{19}$, por lo que cualquier planteamiento terapéutico que se ofrezca debe compararse con 
dichas cifras para permitir a la pareja una elección razonada. Desde nuestro punto de vista, sólo se deberá optar por un tratamiento etiológico en todos aquellos casos en los que concurran las siguientes circunstancias:

- Se haya establecido un diagnóstico etiológico.

- No existan diagnósticos asociados que puedan comprometer el resultado.

- Exista un tratamiento específico.

- No haya un deterioro testicular severo o irreversible.

- Los porcentajes de éxito sean razonables.

- Ausencia de patología concomitante de la mujer.

- Ausencia de patología genética ${ }^{20}$.

- Aceptación por parte de la pareja.

Además habrá que tener en cuenta el deseo de la pareja de tener más hijos en sucesivos embarazos, circunstancia que debería inclinar la elección hacia el tratamiento etiológico.

\subsection{Tratamientos etiológicos y resultados}

Tras instaurar un tratamiento etiológico específico, en nuestra Unidad estamos consiguiendo mejorías significativas del seminograma en más del 50\% de los casos. Se considera mejoría significativa cuando:

- La calidad seminal se incrementa en más de un $50 \%$ (se compara la media de los dos seminogramas previos al tratamiento con la media de al menos tres seminogramas posteriores) y dicho incremento permite recurrir a alguna técnica de reproducción asistida más sencilla que la inicialmente requerida.

- Cuando se produce el embarazo de la pareja.

Globalmente, cuando hemos considerado oportuno realizar un tratamiento etiológico, se ha conseguido un porcentaje de normalización del seminograma superior al 47\%. Los mejores resultados se han obtenido en las siguientes situaciones (Tabla IV):

- Microcirugía reconstructiva de la vía seminal (vaso-vasostomía, epidídimo-vasostomía) ${ }^{21-23}$.

- Corrección de las alteraciones de la erección o eyaculación.

- Infección de la vía seminal.

- Alteraciones hormonales.

- Obstrucción de conductos eyaculadores.

\section{TABLA IV}

RESULTADOS DE LOS TRATAMIENTOS ESPECÍFICOS SEGÚN ETIOLOGÍA

\begin{tabular}{|c|c|c|c|c|c|c|}
\hline & $\begin{array}{c}\text { Número } \\
\text { de } \\
\text { pacientes }\end{array}$ & $\begin{array}{l}\text { Normalización } \\
\text { seminograma }\end{array}$ & $\begin{array}{c}\text { Mejoria } \\
\text { seminograma }\end{array}$ & $\begin{array}{l}\text { Sin cambios } \\
\text { seminograma }\end{array}$ & $\begin{array}{c}\text { Pacientes } \\
\text { perdidos }\end{array}$ & Embarazos \\
\hline $\begin{array}{l}\text { Az. Obstruc. } \\
\text { (Vasectomía) }\end{array}$ & 52 & $38(73,1 \%)$ & $9(17,3 \%)$ & $5(9,6 \%)$ & 9 & 27 (51,9\%) \\
\hline $\begin{array}{l}\text { Az. Obstruc. } \\
\text { (Ob. epididimo) }\end{array}$ & 10 & $2(20 \%)$ & $2(20 \%)$ & $6(60 \%)$ & 2 & $2(20 \%)$ \\
\hline $\begin{array}{l}\text { Az. Obstruc. } \\
\text { (Herniorrafia) }\end{array}$ & 4 & $1(25 \%)$ & $1(25 \%)$ & $2(50 \%)$ & 0 & $1(25 \%)$ \\
\hline Varicocele & 101 & $41(40,6 \%)$ & $36(35,6 \%)$ & $24(23,8 \%)$ & 20 & $26(25,7 \%)$ \\
\hline $\begin{array}{l}\text { Alteración } \\
\text { hormonal }\end{array}$ & 15 & $5(33,3 \%)$ & $5(33,3 \%)$ & $5(33,3 \%)$ & 1 & 3 (20\%) \\
\hline $\begin{array}{l}\text { Alteración } \\
\text { eyaculación }\end{array}$ & 6 & $2(33,3 \%)$ & - & $4(66,6 \%)$ & 1 & $2 / 6$ \\
\hline $\begin{array}{l}\text { Infección } \\
\text { seminal }\end{array}$ & 28 & $12(42,8 \%)$ & $8(28,6 \%)$ & $8(28,6 \%)$ & 3 & $5(17,9 \%)$ \\
\hline $\begin{array}{l}\text { Obstrucción } \\
\text { eyaculadores }\end{array}$ & 14 & $9(64,3 \%)$ & $2(14,3 \%)$ & $3(21,4 \%)$ & 2 & 7 (50\%) \\
\hline Total & 230 & $110(47,8 \%)$ & $63(27,4 \%)$ & $57(23,9 \%)$ & 38 & $73(31,7 \%)$ \\
\hline
\end{tabular}


Resultados superponibles han sido publicados por otros autores ${ }^{21-31}$.

\section{RECUPERACIÓN DE ESPERMATO- ZOIDES PARA FIV-ICSI}

\subsection{Introducción}

La introducción de la técnica de Inyección Intracitoplásmica de Espermatozoides (Intracytoplasmic Sperm Injection o ICSI) por el grupo de Palermo en el año 1992, modificó radicalmente los planteamientos a seguir en los pacientes con azoospermia, necrozoospermia o alteraciones de la eyaculación. En la actualidad, la ausencia de espermatozoides móviles en el eyaculado no impide que el paciente pueda someterse a un procedimiento de FIV-ICSI, consiguiéndose en muchos casos la fertilización ovocitaria con gametos masculinos recuperados directamente del testículo o de la vía seminal (epidídimo o conducto deferente) ${ }^{32,33}$. Estos gametos suelen ser inmóviles, o con escasa movilidad, e inmaduros, pero se ha demostrado ampliamente que ya disponen de capacidad para fecundar al ovocito cuando son microinyectados en su citoplasma.

Las indicaciones para recurrir a las técnicas de recuperación quirúrgica de espermatozoides son las siguientes:

- Azoospermias no obstructivas: la persistencia de pequeños focos en los que se conserva la espermatogénesis permite recuperar espermatozoides en el testículo de algunos de estos pacientes $^{34,35}$.

- Azoospermias obstructivas: independientemente de las causas que la hayan producido. En general se intentará la recuperación de espermatozoides de la porción más distal de la vía seminal en la que se encuentren, siempre que este procedimiento no condicione o limite la capacidad de una reconstrucción microquirúrgica posterior de la misma.

- Alteraciones de la eyaculación: cuando no se puede recurrir o han fracasado otras técnicas aplicables en estos pacientes tales como la recuperación de espermatozoides de la orina (eyaculación retrógrada); procedimientos capaces de provocar la eyaculación (estímulo vibratorio, eléctrico, etc.); tratamientos farmacológicos, etc. ${ }^{36,37}$. En los últimos años resulta cada vez más fre- cuente recurrir directamente a la recuperación quirúrgica de espermatozoides sin haber intentado previamente alguno de los procedimiento anteriormente enumerados.

- Necrozoospermia

\subsection{Técnicas de recuperación quirúrgica de espermatozoides}

Los espermatozoides pueden ser recuperados del propio testículo, del epidídimo o del conducto deferente.

- Recuperación testicular de espermatozoides: puede realizarse mediante dos procedimientos diferentes que son:

- Biopsia quirúrgica abierta: obtención de uno o varios fragmentos de pulpa testicular tras la apertura de las cubiertas testiculares ${ }^{38,39}$.

- Mediante punción-aspiración testicular percutánea: obtención de espermatozoides mediante la punción ciega y percutánea del testículo (procurando evitar dañar el epidídimo o el hilio testicular) y aspiración del mismo. Este tipo de procedimientos plantea un grave riesgo de lesiones testiculares secundarias, fundamentalmente sangrado intraparenquimatoso, que puede agravar aún más el deterioro de la espermatogéne$\operatorname{sis}^{38,40}$

Las indicaciones para la recuperación testicular de espermatozoides del testículo son:

- Azoospermia no obstructiva: en todos los casos ya que la probabilidad de encontrar espermatozoides en el epidídimo o en el conducto deferente es muy remota.

- Azoospermia por obstrucción intratesticular o por ausencia de epidídimo (congénita o adquirida).

- Azoospermia obstructiva, necrozoospermia o alteración de la eyaculación en las que nos se hayan podido recuperar espermatozoides del conducto deferente o del epidídimo.

- Siempre que sea necesario realizar una biopsia testicular por cualquier motivo en caso de azoospermia, necrozoospermia o alteración de la eyaculación.

- Recuperación epididimaria de espermatozoides: Consiste en la punción-aspiración del epidídimo para recuperar los espermatozoides en él acumulados. Según se realice dicha punción hablaremos de: 
- Aspiración percutánea de espermatozoides: punción percutánea y ciega del epidídimo ${ }^{41}$.

- Aspiración macroscópica de espermatozoides del epidídimo: la punción se realiza después de haber practicado una apertura de las cubiertas testiculares hasta llegar al propio epidídimo. Éste se punciona bajo visión directa, eligiendo alguna asa dilatada.

- Aspiración microquirúrgica de espermatozoides del epidídimo: se abren las cubiertas testiculares hasta llegar al epidídimo. Con la ayuda de un microscopio se localiza alguna asa epididimaria dilatada. Se realiza una incisión de ésta, aspirando el contenido de la misma con una micro-pipeta. El asa de epidídimo se cierra posteriormente con la ayuda del microscopio y empleando puntos de nylon de 10/042.

Las indicaciones para realizar una recuperación de espermatozoides del epidídimo serían:

- Obstrucción epididimaria no reparable quirúrgicamente o cuando ha fracasado ésta.

- En casos de agenesia bilateral de conductos deferentes.

- Cuando no se hayan podido recuperar espermatozoides del conducto deferente en casos de obstrucciones distales o alteraciones de la eyaculación.

- Recuperación deferencial de espermatozoides: se trata de recuperar los espermatozoides almacenados en la luz del conducto deferente. Se han descrito dos formas para realizar ésta:

- Aspiración macroscópica del conducto deferente: consiste en la punción directa del conducto deferente, una vez aislado e individualizado, tratando de canalizar su luz.

- Aspiración microscópica del conducto deferente: la aspiración se realiza a través de hemisección transversal en la pared del deferente que, posteriormente, se cierra utilizando microscopio quirúrgico o algún sistema de magnificación de imágenes.

Las indicaciones para proceder a una recuperación de espermatozoides del conducto deferente serían:

- Obstrucción del deferente no solucionable quirúrgicamente o si ha fracasado la reconstrucción.

- Obstrucción de conductos eyaculadores no solucionable mediante cirugía o si ésta ha fracasado previamente.
- Aneyaculación o eyaculación retrógrada cuando no es posible recurrir a otros procedimientos o éstos han fracasado ${ }^{43}$.

\subsection{Resultados en nuestra Unidad}

En nuestra Unidad utilizamos las siguientes técnicas de recuperación de espermatozoides:

- Recuperación de espermatozoides testiculares mediante biopsia abierta. Preferimos esta opción frente a la punción percutánea para evitar el riesgo de daño testicular que se puede ocasionar al realizar múltiples punciones del parénquima. En caso de azoospermia no obstructiva pueden ser necesarias varias incisiones en diferentes zonas del testículo hasta localizar algún foco donde se haya preservado la espermatogénesis.

- Recuperación de espermatozoides del epidídimo mediante punción macroscópica: creemos que es un método sencillo y que ofrece suficientes garantías. La reconstrucción microquirúrgica no pensamos que aporte ninguna ventaja mientras que, por el contrario, incrementa la complejidad técnica, el tiempo quirúrgico y el coste para el paciente.

- Recuperación de espermatozoides mediante punción macroscópica del conducto deferente: La punción y canalización del deferente, sin hemisección del mismo, es un procedimiento muy sencillo, rápido y eficaz. Nuevamente pensamos que la hemisección y reconstrucción microquirúrgica del mismo resulta mucho más laboriosa y costosa, no aportando ninguna ventaja.

Los resultados que hemos obtenido en la recuperación de espermatozoides y en los ciclos de FIV-ICSI han sido los siguientes:

- Ha sido necesaria la recuperación de espermatozoides en 190 pacientes (Tabla V).

- Se han podido recuperar espermatozoides en 157 de los 190 casos $(82,6 \%)$. En las azoospermias obstructivas, necrozoospermias y alteraciones de la eyaculación se han recuperado espermatozoides en el 100\% de los pacientes. En las azoospermias no obstructivas este porcentaje se reduce hasta el 54,2\% $(39 / 72)^{34}$.

- La recuperación se realizó mediante biopsia testicular en 117 casos $(61,6 \%)$; aspiración epididimaria en 15 casos $(7,9 \%)$ o mediante aspiración deferencial en 58 casos $(30,5 \%)$ (Tabla VI). 
TABLA V

PACIENTES EN LOS QUE HA SIDO NECESARIO RECUPERACIÓN QUIRÚRGICA DE ESPERMATOZOIDES (RQE)

\begin{tabular}{||c|c|c|}
\hline \multirow{3}{*}{$\begin{array}{c}\text { Azoospermias } \\
\text { obstructivas } \\
(99)\end{array}$} & Malformación congénita & 2 \\
\cline { 2 - 3 } & Obstrucción epididimaria & 21 \\
\cline { 2 - 3 } & Vasectomía previa & 62 \\
\cline { 2 - 3 } & Herniorrafia bilateral & 6 \\
\hline \multirow{2}{*}{$\begin{array}{c}\text { Azoospermias } \\
\text { no obstructivas } \\
(72)\end{array}$} & ABCD & 8 \\
\cline { 2 - 3 } & Idiopática & 54 \\
\hline \multirow{2}{*}{$\begin{array}{c}\text { Alt. eyaculación } \\
(11)\end{array}$} & Post RT o QT & 6 \\
\cline { 2 - 3 } & Aneyaculación & 5 \\
\hline $\begin{array}{c}\text { Necrozoospermia } \\
(8)\end{array}$ & Necrozoospermia idiopática & 8 \\
\hline Total & & 12 \\
\hline \hline
\end{tabular}

TABLA VI

INDICACIONES PARA LA REALIZACIÓN DE LAS DIFERENTES TÉCNICAS DE RQE

\begin{tabular}{|c|c|c|c|}
\hline \multirow{5}{*}{$\begin{array}{l}\text { RQE mediante } \\
\text { biopsia } \\
\text { testicular }\end{array}$} & \multirow{5}{*}{117} & Azoospermia no obstructiva & 72 \\
\hline & & Necrozoospermia & 6 \\
\hline & & Azoospermia obstructiva & 34 \\
\hline & & Eyaculación retrógrada & 3 \\
\hline & & Aneyaculación & 2 \\
\hline \multirow{3}{*}{$\begin{array}{l}\text { RQE mediante } \\
\text { aspiración } \\
\text { epididimaria }\end{array}$} & \multirow{3}{*}{15} & $\mathrm{ABCD}$ & 5 \\
\hline & & Vasectomía previa & 2 \\
\hline & & Obstrucción epidídimo & 8 \\
\hline \multirow{6}{*}{$\begin{array}{l}\text { RQE mediante } \\
\text { aspiración } \\
\text { deferencial }\end{array}$} & \multirow{6}{*}{58} & Herniorrafia previa & 3 \\
\hline & & Malformación congénita & 2 \\
\hline & & Vasectomía previa & 45 \\
\hline & & Aneyaculación & 3 \\
\hline & & Eyaculación retrógrada & 3 \\
\hline & & Necrozoospermia & 2 \\
\hline
\end{tabular}

- La tasa de embarazo por ciclo en casos de recuperación de espermatozoides fue del $32,8 \%$. No se han encontrado diferencias significativas entre esta tasa de embarazo y la tasa global para FIV-ICSI de nuestra Unidad $(34,9 \%)$.

\section{SCREENING ANDROLÓGICO PREVIO A FIV-ICSI}

\subsection{Introducción; indicaciones para scree-} ning andrológico

En todas aquellas parejas candidatas a FIVICSI por un factor masculino severo (oligoastenozoospermia severa o azoospermia), antes de iniciar el ciclo propiamente dicho, deberían realizarse una serie de estudios encaminados a:

- Establecer un diagnóstico etiológico en el varón, siempre que sea posible (ver capítulo $\mathrm{n}^{\circ}$ $2)^{6,7}$.

- Valorar la posible existencia y oportunidad de un tratamiento etiológico que permita la mejoría e incluso la normalización del seminograma (ver capítulo $\left.\mathrm{n}^{\circ} 2\right)^{6,44-46}$.

- Valorar la posible existencia de una alteración genética, cuya incidencia es muy elevada en este grupo de pacientes ${ }^{47-51}$.

- Descartar posibles factores capaces de reducir los porcentajes de éxito de la TRA, fundamentalmente el bloqueo de la meiosis, las alteraciones en la dotación cromosómica de los espermatozoides y la infección de la vía seminal $^{52}$.

\subsubsection{Descartar posibles patologias gené- ticas transmisibles}

En las parejas candidatas a procedimientos de FIV o FIV-ICSI por factor masculino severo, debería descartarse la existencia de determinadas patologías de carácter genético ${ }^{53}$. La importancia de este diagnóstico es triple:

- En primer lugar, porque la prevalencia de estas alteraciones es muy elevada entre los casos de factor masculino severo (y esta prevalencia es tanto mayor cuanto más severo es el deterioro del seminograma) ${ }^{54}$.

- En segundo lugar, hay que tener en cuenta que en un elevado porcentaje de estos casos va a ser posible la descendencia recurriendo a procedimientos de FIV-ICSI, pudiendo trasmitirse a los hijos las alteraciones genéticas de las que el padre es portador ${ }^{50,55}$.

- Por último, la existencia de alguna de estas anomalías genéticas puede condicionar una reducción significativa en los porcentajes de éxito de las propias técnicas de reproducción asistida y un incremento en el número de abortos ${ }^{56}$. 
El conocimiento de dichas situaciones tiene, por tanto, un gran interés desde el punto de vista clínico, pronóstico e incluso legal. Los estudios genéticos necesarios son enumerados a continuación, añadiendo los resultados obtenidos con nuestros pacientes.

- Estudio de mutaciones del gen CFTR y del alelo $5 T$

En casos de agenesia uni o bilateral de conductos deferentes (ABCD; AUCD). La ABCD y probablemente también la AUCD podrían ser una forma leve (con afectación limitada a la vía seminal) de fibrosis quística (FQ). En estos pacientes es imprescindible reconocer la existencia de mutaciones del gen CFTR (Cystic Fibrosis Transmembrane Regulator) así como la presencia del alelo 5T (existencia de politimidina en el intrón 8, lo cual podría suponer que se manifestara la enfermedad incluso en heterozigosis), cuantificando el riesgo de padecer una fibrosis quística (FQ) por parte del embrión engendrado y proporcionando el consejo genético oportuno ${ }^{57,58}$. Habitualmente, se realiza sólo un estudio de las mutaciones más frecuentes en la población a los dos miembros de la pareja ${ }^{59,60}$.

En nuestra Unidad se han encontrado 9 casos de varones con $\mathrm{ABCD}(1,8 \%)$ y 9 de AUCD $(2,1 \%)$. De los 9 varones con $\mathrm{ABCD}, 4$ eran portadores de alguna mutación del gen CFTR $(44,4 \%)$, en un caso la mujer también era portadora. Por lo que respecta a las AUCD, de los 9 casos, en 5 se encontró una agenesia renal y ureteral ipsilateral, ninguno de los cuales era portador de mutaciones; en 2 casos el estudio realizado era normal y los 2 restantes eran portadores de alguna mutación del gen CFTR $(22,2 \%)$.

Microdelecciones (delección del gen DAZ, regiones $A Z F a, A Z F b, A Z F c)$ en el brazo largo del cromosoma $Y(Y q)$

Muchas de las azoospermias u oligozoospermias severas de carácter secretor son debidas a la existencia de este tipo de alteración genética ${ }^{61-63}$. En nuestra serie, se estudiaron 51 varones con azoospermia u OTA severa, encontrándose una microdelección en 9 de ellos $(17,6 \%)^{64,65}$.

\section{- Anomalias cromosómicas somáticas}

Este tipo de anomalías son detectables en el cariotipo. Su incidencia es de alrededor del 5\%, cifra que resulta unas diez veces superior a la encontrada en la población general ${ }^{66-70}$. En todos los pacientes con azoospermia, OTA severa, abortos de repetición, antecedentes personales o familiares de cromosomopatía y antecedentes personales de exposición a radio o quimioterapia debe realizarse un cariotipo. Nosotros hemos realizado un cariotipo a un total de 283 pacientes, encontrando alteraciones en 13 de ellos (4,6\%). Las anomalías encontradas fueron:

- Síndrome de Klinefelter (47 XXY): 5 pacientes 47 XYY: 2 pacientes.

- Mosaico $46 \mathrm{XY} / 45 \mathrm{XO}$ : 1 paciente.

- Traslocación Robertsoniana 46 XY t(14;13): 1 paciente.

- Traslocación recíproca $46 \mathrm{XY}$ t(12;11): 1 paciente.

- Macrodelección del cromosoma Y (g11.23qter): 1 paciente.

- 7q11;23/ Sindrome de William: 46 XY t (11;14): 1 paciente.

- Traslocación equilibrada 46 XY t $(1,17)$ (q21,q25): 1 paciente.

- Anomalías del apareamiento cromosómico durante el proceso de meiosis

Origen de alteraciones numéricas (diploides o aneuploides) en la dotación cromosómica del espermatozoide ${ }^{71-74}$. Las indicaciones para realizar este tipo de estudios serian: azoospermia, OTA severa, abortos de repetición, fallos de ciclos previos de FIV y existencia de anomalías cromosómicas somáticas (conocer su comportamiento meiótico). Hemos realizado este tipo de estudio a un total de 158 pacientes, encontrando anomalías del apareamiento de los cromosomas homólogos en 12 de ellos $(7,6 \%)$.

\subsubsection{Diagnóstico de factores de mal pro- nóstico}

Se trata de descartar la existencia de patologías que reducen de una manera significativa las posibilidades de éxito de las técnicas de reproducción asistida. En este sentido las principales alteraciones son: 
- Infecciones de la vía seminal y contaminación secundaria de las muestras de semen

La contaminación bacteriana de las muestras de semen, independientemente de su etiología, va a condicionar una clara disminución en los porcentajes de éxito de las TRA, siendo causa frecuente de fallos de fecundación, embriones de mala calidad, abortos precoces, etc. ${ }^{75}$. Por todo ello, ante la sospecha de un semen con infección se deberian realizar los estudios pertinentes, encaminados a su diagnóstico y posterior tratamiento ${ }^{76}$. En aquellos casos en los que no sea posible erradicar la infección, se debe administrar un tratamiento antibiótico oportuno previo a la recolección de la muestra de semen a utilizar en el ciclo de FIV $^{77}$.

En nuestra Unidad hemos encontrado 35 casos de infección de la vía seminal demostrable mediante cultivo fraccionado de orina y semen. De ellos, 30 fueron estudiados por deterioro de la calidad seminal (OTA) y 5 por fracaso de varios ciclos previos de FIV sin deterioro llamativo en el seminograma. La infección se consiguió erradicar mediante tratamiento antibiótico oportuno (establecido por antibiograma) en 31 pacientes. En los

\section{TABLA VII}

EMBARAZOS POR CICLO FIV EN FUNCIÓN DE LA EXISTENCIA O NO DE BLOQUEO DE LA MEIOSIS ( $<<0,001)$

\begin{tabular}{|l|c|c|c|}
\hline & $\begin{array}{c}\text { Bloqueo } \\
\text { meiosis }\end{array}$ & $\begin{array}{c}\text { Serie } \\
\text { general }\end{array}$ & Total \\
\hline Embarazo & $17(25 \%)$ & $177(33,7 \%)$ & 194 \\
\hline $\begin{array}{l}\text { Embarazo } \\
\text { bioquímico }\end{array}$ & $5(7,3 \%)$ & $30(5,7 \%)$ & 35 \\
\hline No embarazo & $46(67,6 \%)$ & $318(60,6 \%)$ & 364 \\
\hline Total & 68 & 525 & 593 \\
\hline
\end{tabular}

TABLA VIII

ABORTOS POR EMBARAZO SEGÚN HAYA O NO BLOQUEO DE LA MEIOSIS ( $p<0,001)$

\begin{tabular}{|l|c|c|c|}
\hline & $\begin{array}{c}\text { Bloqueo } \\
\text { meiosis }\end{array}$ & $\begin{array}{c}\text { Serie } \\
\text { general }\end{array}$ & Total \\
\hline Aborto & $8(47,1 \%)$ & $17(9,6 \%)$ & 25 \\
\hline No aborto & $9(52,9 \%)$ & $160(90,4 \%)$ & 169 \\
\hline Total & 17 & 177 & 194 \\
\hline
\end{tabular}

otros 4 no se consiguió su erradicación a pesar de instaurar ciclos sucesivos de tratamiento antibiótico.

- Existencia de bloqueos severos de la meiosis

Hemos realizado un total de 158 estudios citogenéticos de meiosis, obteniendo los siguientes resultados: estudio normal en 92 casos $(58,2 \%)$; bloqueo severo de la meiosis en 54 $(34,2 \%)$ y anomalías de apareamiento cromosómico en $12(7,6 \%)$. Cada vez son más numerosos los estudios que demuestran la relación entre fracaso de las técnicas de reproducción asistida (TRA) y la existencia de bloqueos severos de la meiosis ${ }^{72,73,78}$. En nuestra serie hemos encontrado una reducción significativa en los porcentajes de embarazo así como un incremento muy sustancial en el porcentaje de abortos cuando el varón presenta este tipo de anomalía de la meiosis (Tablas VII y VIII).

\section{REFERENCIAS}

1. DUCKITT K.: Infertility and subfertility. Clin Evid 2002; (7): 1679-1704.

2. DEVOTO E, MADARIAGA M, LIOI X.: Causes of male infertility. The contribution of the endocrine factor. Rev Med Chil 2000; 128 (2): 184-192.

3. WONG WY, THOMAS CM, MERKUS JM, ZIELHUIS GA, STEEGERS-THEUNISSEN RP.: Male factor subfertility: possible causes and the impact of nutritional factors. Fertil Steril 2000; 73 (3): 435-442.

4. SANDLOW JI.: Shattering the myths about male infertility. Treatment of male factors may be more successful and cost-effective than you think. Postgrad Med 2000; 107 (2): 235-239, 242, 245.

5. MARTIN-DU-PAN R.: Do viagra and fertilization in vitro announce the end of etiologic treatments in andrology?. J Gynecol Obstet Biol Reprod (Paris) 2000; 29 (4): 345-349.

6. SANDLOW JI.: Shattering the myths about male infertility. Treatment of male factors may be more successful and cost-effective than you think. Postgrad Med 2000; 107 (2): 235-239, 242, 245.

7. DICKEY RP, TAYLOR SN, RYE PH, LU PY, SARTOR BM.: Infertility is a symptom, not a disease. Fertil Steril 2000; 74 (2): 398.

8. KOLETTIS PN, SABANEGH ES.: Significant medical pathology discovered during a male infertility evaluation. J Urol 2001; 166 (1): 178-180.

9. KURILO LF, SHILEIKO LV, SOROKINA TM, GRISHINA EM.: Pattern of hereditary reproductive disorders. Vestn Ross Akad Med Nauk 2000; (5): 32-36.

10. KOLETTIS PN, SABANEGH ES.: Significant medical pathology discovered during a male infertility evaluation. J Urol 2001; 166 (1): 178-180.

11. BRUNEREAU L, FAUCHIER F, FERNANDEZ P et al. Sonographic evaluation of human male infertility. $J$ Radiol 2000; 81 (12): 1693-1701. 
12. YASSA NA, KEESARA S.: Role of transrectal ultrasonography in evaluating the cause of azoospermia. Can Assoc Radiol J 2001; 52 (4): 266-268.

13. BUKHARIN OV, KUZ'MIN MD, IVANOV IUB.: The role of the microbial factor in the pathogenesis of male infertility. Zh Mikrobiol Epidemiol Immunobiol 2000; (2): 106-110.

14. SILVA PD, LARSON KM, VAN EVERY MJ, SILVA DE.: Successful treatment of retrograde ejaculation with sperm recovered from bladder washings. A report of two cases. J Reprod Med 2000; 45 (11): 957-960.

15. YOGEV L, GAMZU R, KLEIMAN S, BOTCHAN A, HAUSER R, YAVETZ H.: Evaluation of meiotic impairment of azoospermic men by fluorescence in situ hybridization. Fertil Steril 2000; 74 (2): 228-233.

16. LELLEI I, MAGYAR E, ERDEI E.: Histological evaluation of multiple testicular biopsies helping assisted reproduction. Pathol Res Pract 2001; 197 (11): 727 733.

17. ARIBARG A, NGEAMVIJAWAT J, CHANPRASIT Y.: Investigation of sex chromosome abnormalities in teratozoospermia of infertile men using fluorescence in situ hybridization. J Med Assoc Thai 2000; 83 (7): 737-742.

18. LELLEI I, MAGYAR E, ERDEI E.: Histological evaluation of multiple testicular biopsies helping assisted reproduction. Pathol Res Pract 2001; 197 (11): 727 733.

19. OEHNINGER S.: Place of intracytoplasmic sperm injection in management of male infertility. Lancet 2001; 357 (9274): 2068-2069.

20. CAYAN S, LEE D, BLACK LD, REIJO PERA RA, TUREK PJ.: Response to varicocelectomy in oligospermic men with and without defined genetic infertility. Urology 2001; 57 (3): 530-535.

21. MATSUDA T.: Microsurgical epididymovasostomy. Int J Urol 2000; 7 Suppl: S39-S41.

22. HEIDENREICH A, ALTMANN P, NEUBAUER S ENGELMANN UH.: Microsurgical vasovasostomy in the age of modern reproduction medicine. A cost-benefit analysis. Urologe A 2000; 39 (3): 240-245.

23. HEIDENREICH A, ALTMANN P, ENGELMANN UH.: Microsurgical vasovasostomy versus microsurgical epididymal sperm aspiration/testicular extraction of sperm combined with intracytoplasmic sperm injection. A cost-benefit analysis. Eur Urol 2000; 37 (5): 609-614.

24. LIGUORI G, BUCCI S, PAOLINELLI D et al.: Minimally invasive treatment of intraprostatic cysts. Arch Ital Urol Androl 2000; 72 (4): 357-360.

25. OZGOK Y, TAN MO, KILCILER M, TAHMAZ L, KIBAR Y.: Diagnosis and treatment of ejaculatory duct obstruction in male infertility. Eur Urol 2001; 39 (1): 24-29.

26. PAVLOVICH CP, KING P, GOLDSTEIN M, SCHLEGEL PN.: Evidence of a treatable endocrinopathy in infertile men. J Urol 2001; 165 (3): 837-841.

27. PERIMENIS P, MARKOU S, GYFTOPOULOS K, ATHANASOPOULOS A, BARBALIAS G.: Effect of subinguinal varicocelectomy on sperm parameters and pregnancy rate: a two-group study. Eur Urol 2001; 39 (3): 322-325.

28. SCHOOR RA.: Prostatitis and male infertility: evidence and links. Curr Urol Rep 2002; 3 (4): 324-329.

29. SCHROEDER-PRINTZEN I, LUDWIG M, KOHN F, WEIDNER W.: Surgical therapy in infertile men with ejaculatory duct obstruction: technique and outcome of a standardized surgical approach. Hum Reprod 2000; 15 (6): 1364-1368.
30. SILBER SJ.: Evaluation and treatment of male infertility. Clin Obstet Gynecol 2000; 43 (4): 854-888.

31. SUN GH, LEE SS, YU DS, CHEN HI, CHANG SY.: Successful treatment of azoospermia secondary to ejaculatory duct cyst. Arch Androl 2000; 45 (1): 25-28.

32. OEHNINGER S.: Place of intracytoplasmic sperm injection in management of male infertility. Lancet 2001; 357 (9274): 2068-2069.

33. WINDT ML, COETZEE K, KRUGER TF, MENKVELD R, VAN MJ.: Intracytoplasmic sperm injection with testicular spermatozoa in men with azoospermia. $J$ Assist Reprod Genet 2002; 19 (2): 53-59.

34. AMER M, ATEYAH A, ZOHDY W, ABD EL NASSER T, ABDEL-MALAK G, FAKHRY E.: Preoperative and intraoperative factors that predict difficult testicular sperm retrieval in patients with nonobstructive azoospermia. Fertil Steril 2002; 78 (3): 646-647.

35. CHAN PT, SCHLEGEL PN.: Nonobstructive azoospermia. Curr Opin Urol 2000; 10 (6): 617-624.

36. BIERING-SORENSEN F, SONKSEN J.: Sexual function in spinal cord lesioned men. Spinal Cord 2001; 39 (9): 455-470.

37. OHL DA, WOLF LJ, MENGE AC et al. Electroejaculation and assisted reproductive technologies in the treatment of anejaculatory infertility. Fertil Steril 2001; 76 (6): 1249-1255.

38. RIBE N, MANASIA P, MARTINEZ L, EGOZCUE S, POMEROL JM.: TESE-ICSI in the treatment of secretory azoospermia secondary to cryptorchism. Report of a clinical case. Arch Ital Urol Androl 2001; 73 (1): 45-48.

39. SCHOOR RA, ELHANBLY S, NIEDERBERGER CS, ROSS LS.: The role of testicular biopsy in the modern management of male infertility. J Urol 2002; 167 (1): 197-200.

40. TUREK PJ, LJUNG BM, CHA I, CONAGHAN J.: Diagnostic findings from testis fine needle aspiration mapping in obstructed and nonobstructed azoospermic men. J Urol 2000; 163 (6): 1709-1716.

41. CHATTERJEE S, CHAUDHURI AR, CHOWDHURI RG, RAHMAN S.: Percutaneous epididymal sperm aspiration followed by intrauterine insemination-a new approach to achieve a pregnancy in infertility due to obstructive azoospermia. J Indian Med Assoc 2001; 99 (8): 445-447.

42. OKADA H, DOBASHI $M$, YAMAZAKI $T$ et al. Conventional versus microdissection testicular sperm extraction for nonobstructive azoospermia. J Urol 2002; 168 (3): 1063-1067.

43. BRACKETT NL, LYNNE CM, ABALLA TC, FERRELL SM.: Sperm motility from the vas deferens of spinal cord injured men is higher than from the ejaculate. $J$ Urol 2000; 164 (3 Pt 1): 712-715.

44. JAROW JP, SHARLIP ID, BELKER AM et al.: Best practice policies for male infertility. J Urol 2002; 167 (5): 2138-2144.

45. SILBER SJ.: Evaluation and treatment of male infertility. Clin Obstet Gynecol 2000; 43 (4): 854-888.

46. SILBER SJ.: New concepts in operative andrology: a review. Int $J$ Androl 2000; 23 Suppl 2: 66-76.

47. ANTONELLI A, GANDINI L, PETRINELLI $\mathrm{P}$ et al.: Chromosomal alterations and male infertility. $J$ Endocrinol Invest 2000; 23 (10): 677-683.

48. LUDWIG M, GEIPEL A, BERG C, GEMBRUCH U, SCHWINGER E, DIEDRICH K.: Is intracytoplasmic sperm injection itself an indication to perform preimplantation genetic diagnosis (PGD)? About PGD, invasive prenatal diagnosis and genetic sonography. Fetal Diagn Ther 2001; 16 (2): 68-82. 
49. MACAS E, IMTHURN B, KELLER PJ.: Increased incidence of numerical chromosome abnormalities in spermatozoa injected into human oocytes by ICSI Hum Reprod 2001; 16 (1): 115-120.

50. MIKKELSEN AL, LIDEGAARD O, MELDGAARD M BRONDUM-NIELSEN K, LINDENBERG S.: Severe male infertility. Genetic investigation and counselling prior to intracytoplasmic sperm injections. Lakartidningen 2000; 97 (11): 1269-1272.

51. TUERLINGS JH, KREMER JA.: Genetics of male factor subfertility. Eur J Obstet Gynecol Reprod Biol 2000; 89 (2): 117-121.

52. CALOGERO AE, DE PALMA A, GRAZIOSO C et al.: High sperm aneuploidy rate in unselected infertile patients and its relationship with intracytoplasmic sperm injection outcome. Hum Reprod 2001; 16 (7): 1433-1439.

53. LEVRON J, AVIRAM-GOLDRING A, MADGAR I, RAVIV G, BARKAI G, DOR J.: Sperm chromosome abnormalities in men with severe male factor infertility who are undergoing in vitro fertilization with intracytoplasmic sperm injection. Fertil Steril 2001; 76 (3): 479-484.

54. WOJDA A, KORCZ A, JEDRZEJCZAK $\mathrm{P}$ et al: Importance of cytogenetic analysis in patients with azoospermia or severe oligozoospermia undergoing in vitro fertilization. Ginekol Pol 2001; 72 (11): 847-853.

55. CRAM DS, MA K, BHASIN S et al.: Y chromosome analysis of infertile men and their sons conceived through intracytoplasmic sperm injection: vertical transmission of deletions and rarity of de novo deletions. Fertil Steril 2000; 74 (5): 909-915.

56. EGOZCUE S, BLANCO J, VENDRELL JM et al.: Human male infertility: chromosome anomalies, meiotic disorders, abnormal spermatozoa and recurrent abortion. Hum Reprod Update 2000; 6 (1): 93-105.

57. PHILLIPSON GT, PETRUCCO OM, MATTHEWS CD. Congenital bilateral absence of the vas deferens, cystic fibrosis mutation analysis and intracytoplasmic sperm injection. Hum Reprod 2000; 15 (2): 431-435.

58. STUHRMANN M, DORK T.: CFTR gene mutations and male infertility. Andrologia 2000; 32 (2): 71-83.

59. LEWIS-JONES DI, GAZVANI MR, MOUNTFORD R.: Cystic fibrosis in infertility: screening before assisted reproduction: opinion. Hum Reprod 2000; 15 (11): 2415-2417.

60. LOTEM Y, BARAK A, MUSSAFFI H et al.: Reaching the diagnosis of cystic fibrosis-the limits of the spectrum. Isr Med Assoc J 2000; 2 (2): 94-98.

61. DOHLE GR, HALLEY DJ, VAN HEMEL JO et al. Genetic risk factors in infertile men with severe oligozoospermia and azoospermia. Hum Reprod 2002; 17 (1): 13-16.

62. FERNANDES S, HUELLEN K, GONCALVES $\mathrm{J}$ et al.: High frequency of DAZ1/DAZ2 gene deletions in patients with severe oligozoospermia. Mol Hum Reprod 2002; 8 (3): 286-298.

63. FORESTA C, FERLIN A, MORO E, MARIN P, ROSSI A, SCANDELLARI C.: Microdeletion of chromosome Y in male infertility: role of the DAZ gene. Ann Ital Med Int 2001; 16 (2): 82-92.

64. KRAUSZ C, QUINTANA-MURCI L, MCELREAVEY K.: Prognostic value of Y deletion analysis: what is the clinical prognostic value of Y chromosome microdeletion analysis? Hum Reprod 2000; 15 (7): 1431-1434.
65. MARTÍNEZ MC, BERNABÉ MJ, GÓMEZ E y cols.: Screening for AZF deletion in a large series of severely impaired spermatogenesis patients. J Androl 2000; 21 (5): 651-655.

66. NAKAMURA Y, KITAMURA M, NISHIMURA K et al.: Chromosomal variants among 1790 infertile men. Int J Urol 2001; 8 (2): 49-52.

67. OGAWA S, ARAKI S, ARAKI Y, OHNO M, SATO I.: Chromosome analysis of human spermatozoa from an oligoasthenozoospermic carrier for a 13;14 Robertsonian translocation by their injection into mouse oocytes. Hum Reprod 2000; 15 (5): 1136-1139.

68. PENNA VIDEAU S, ARAUJO $H$, BALLESTA $F$, BALLESCA JL, VANRELL JA.: Chromosomal abnormalities and polymorphisms in infertile men. Arch Androl 2001; 46 (3): 205-210.

69. RUBIO C, GIL-SALOM M, SIMON C y cols.: Incidence of sperm chromosomal abnormalities in a risk population: relationship with sperm quality and ICSI outcome. Hum Reprod 2001; 16 (10): 2084-2092.

70. VINCENT MC, DAUDIN M, DE MP et al.: Cytogenetic investigations of infertile men with low sperm counts: a 25- year experience. $J$ Androl 2002; 23 (1): 18-22; discussion 44-45.

71. PATRIZIO P, RICCI SM, TOMASZEWSKI JE, HECHT NB.: Identification of meiotic and postmeiotic gene expression in testicular tissue of patients histologically classified as Sertoli cell only. Fertil Steril 2000; 74 (4): 785-790.

72. EGOZCUE S, BLANCO J, VENDRELL JM et al. Human male infertility: chromosome anomalies, meiotic disorders, abnormal spermatozoa and recurrent abortion. Hum Reprod Update 2000; 6 (1): 93-105.

73. EGOZCUE S, VENDRELL JM, GARCIA $\mathrm{F}$ y cols.: Increased incidence of meiotic anomalies in oligoasthenozoospermic males preselected for intracytoplasmic sperm injection. J Assist Reprod Genet 2000; 17 (6): 307-309.

74. EGOZCUE S, BLANCO J, VIDAL F, EGOZCUE J.: Diploid sperm and the origin of triploidy. Hum Reprod 2002; 17 (1): 5-7.

75. LUDWIG M, DIMITRAKOV J, DIEMER T, HUWE P, WEIDNER W. Prostatitis syndrome. Changes in the ejaculate and effects on fertility. Urologe A 2001; 40 (1): 18-23.

76. CHAN PT, SCHLEGEL PN.: Inflammatory conditions of the male excurrent ductal system. Part II. J Androl 2002; 23 (4): 461-469.

77. VICARI E.: Effectiveness and limits of antimicrobial treatment on seminal leukocyte concentration and related reactive oxygen species production in patients with male accessory gland infection. Hum Reprod 2000; 15 (12): 2536-2544.

78. NUDELL D, CASTILLO M, TUREK PJ, PERA RR.: Increased frequency of mutations in DNA from infertile men with meiotic arrest. Hum Reprod 2000; 15 (6): 1289-1294.

Dr. I. Galmés Belmonte

Po de la Castellana, $129-7^{\circ} \mathrm{B}$

28046 Madrid

(Trabajo recibido el 25 noviembre de 2003) 